\title{
Assessing the Potential of FT-IR to Identify Clinical Strains of Candida spp.
}

\author{
Filipa Brandão, Cristina Veríssimo and João Brandão*
}

\author{
National Institute of Health Dr. Ricardo Jorge, Department of Infectious Diseases, Av. Padre Cruz 1649-016 Lisboa, \\ Portugal
}

\begin{abstract}
Candida spp were examined by FT-IR in order to compare identification using FT-IR with biochemical identification (bioMerieux ${ }^{\mathbb{B}}$ ). FT-IR revealed itself as a fast and effective method for identification of the strains tested as all were placed into the appropriate species, including one which was misidentified by the biochemical method.
\end{abstract}

Keywords: Candida, FT-IR.

\section{INTRODUCTION}

Fungi constitute a much diversified taxonomic group of organisms. Over 100,000 species have been described, most of which exist outside aquatic environments. Morphological differences form the basis for classical identification systems. This method is emphatically deficient due to a lack of standardization and subjectivity, as the spectra obtained vary with culture conditions and age and the scientific community is still seeking best performing frequency ranges and analysis to use. Many phenotypic characteristics are unstable and too dependent on growth conditions. However, Roscini et al. demonstrated that the analysis of an average of 5 replicas of each spectrum for a strain is sufficient to eliminate most of the variations observed individually, thus reducing the background noise when comparing strains [1]. Also the region of the infra-red spectra to use can be manipulated in order to enhance the differences between strains by choosing regions related mainly to RNA and Proteins - RNA (ca $1080 \mathrm{~cm}-1$ ) and proteins (amide II ca $1540 \mathrm{~cm}-1)$ [2]. Another limitation to this method is the inability to apply it to fungi that can't be cultured. Yet, many fungi were not identified correctly until alternative methods (based on genetics or spectroscopic characteristics) were applied [3].

Molecular methods, which were initially an activity bound to research, are becoming increasingly common in routine fungal identifications. These techniques provide knowledge that duel the traditional concept of species and generated phylogenetic classification schemes [3]. Population and molecular data show that this group is constituted by various unrelated taxa. Their true nature is hence being revealed by non-morphological techniques. Spectroscopy has provided a more recent type of physicalchemical identi-fication method called "whole-organism fingerprinting" [4].

\footnotetext{
*Address correspondence to this author at the National Institute of Health Dr. Ricardo Jorge, Department of Infectious Diseases, Av. Padre Cruz 1649016 Lisboa, Portugal; Tel: (+351) 217.519.247; Fax: (+351) 217.526.400; E-mail: joao.brandao@insa.min-saude.pt
}

Infra-red (IR) spectroscopy is based on the excitation of molecular bonds. Each bond absorbs a specific wave-length within the IR spectra, revealing a specific fingerprint for each molecule generated by the sum of the absorption of all atom-atom bonds [1]. Many advanced spectrophotometers scan ranges IR radiation, through the means of an interferometer use together with Fourrier mathematical analysis of the raw data.

An IR spectrum with Fourier Transformation (FT-IR) of a microorganism constitutes a highly specific biochemical fingerprint. This process is a fast, non-destructive analytical method and the fingerprint is reproducible $[4,5]$ allowing the rapid distinction of genera and species with low cost from a small amount of analytical biomass $[5,6]$. It also allows the phonotypical typing and characterization of microbial species, strains, biochemical behaviors and adaptation mechanisms and can even be used to identify certain macromolecules (nature, quantity and conformation) present in the cells [5]. A fast response is important for the medical community, especially when considering that Candida is the $4^{\text {th }}$ most frequently isolated microorganism from nosocomial infections [7].

The FT-IR spectra may reflect variations due to culturing parameters (such as composition of the growth media, $\mathrm{pH}$, temperature, humidity, storage method, etc.) $[5,6]$ which may be resolved with their standardization and growth in controlled environment. This is in fact very important for the reproducibly of the spectroscopic analysis of microorganisms [5].

Our lab decided to test this approach as a means to assess possible outbreaks, given the fine results when comparing two strains.

\section{METHODS}

21 strains of Candida spp were randomly (blindly) collected out of clinical isolates of the Mycology laboratory of the National Institute of Health Dr. Ricardo Jorge (nonsystemic infections) and identified with the Biomerieux ${ }^{B}$ ID32C system. FT-IR was performed on all 21 strains, following growth at $35+/-2^{\circ} \mathrm{C}$ during $24+/-1$ hours in 
gelified Sabouraud medium with cloramphenicol added. 35 microliters of a suspension of each strain in distilled water $(\mathrm{OD} 500=3)$ were deposited on a well of the FT-IR plate, followed by drying at $44+/-0.5^{\circ} \mathrm{C}$ for $45+/-5$ minutes. Scans were made in a Bruker ${ }^{\circledR}$ FT-IR spectrophotometer (in the HTS-XT module), of the region of 500 to $2000 \mathrm{~cm}^{-1}$ and with the aid of Ward's algorithm and base-line corrections, dendrograms of the second derivatives of the spectrophotometer's output were built by Opus (Bruker ${ }^{(B)}$ ) as described in Roscini, L. et al. [1]. Seven replicates of each sample were run but only green results were used for the building the dendrogram and subsequent comparison of identification with biochemical identification

\section{RESULTS AND DISCUSSION}

Table 1 reveals the biochemical identification and the number of green wells (quality approved by the software of the equipment) out of seven obtained in one run of FT-IR analysis for each strain.

Most of the isolates are $C$ albicans, which is consistent with the findings of our lab in routine analysis. However we did obtain other species which was a must in order to be able to compare FT-IR results of a different species and strains of the same species. In this batch of 21 strains, in one run, we only had 3 strains that produced 7 acceptable replicas (green wells). For 4 strains only 1 well was accepted. Overall the rejection rate (red wells) was $47 \%$. Fig. (1) shows the dendrogram and identification results together and reveals that it is possible to use FT-IR to group up strains of different species, following the method described in materials and methods.

The maximum heterogeny found is the separation of the C. albicans/ C. lipolytica cluster and the others species (5.5) C. lipolytica clustered with $C$. albicans. With one usable well, it's impossible to tell if all $C$. lipolytica replicates would cluster together or if they would be scattered throughout the $C$. albicans cluster. The strain is however not a mis-identified $C$. albicans (confirmed by molecular fingerprints of random amplification using GACA - data notshown).

From the n-tuplets analysis it's possible to set the low heterogeny cut-off at 0.5 units, the highest value found within replicas. This means that no significant difference between two strains is to be considered between 0 and this value, or so we assumed. This however indicates that the clusters of the species obtained in this analysis are significant.

Strain 59, although identified as $C$. albicans has in fact been clustered with other $C$. parapsilosis (the 4 last strains on the right in Fig. 1). This was been confirmed molecularly also using GACA fingerprints where the patterns are consistent with those of that species and not of $C$ albicans (data not-shown). We have no explanation for this fact but it suggests that the IR region used in this work might be

Table 1. Identification Results and Number of Green (Usable) Wells of FT-IR for Each Strain Tested

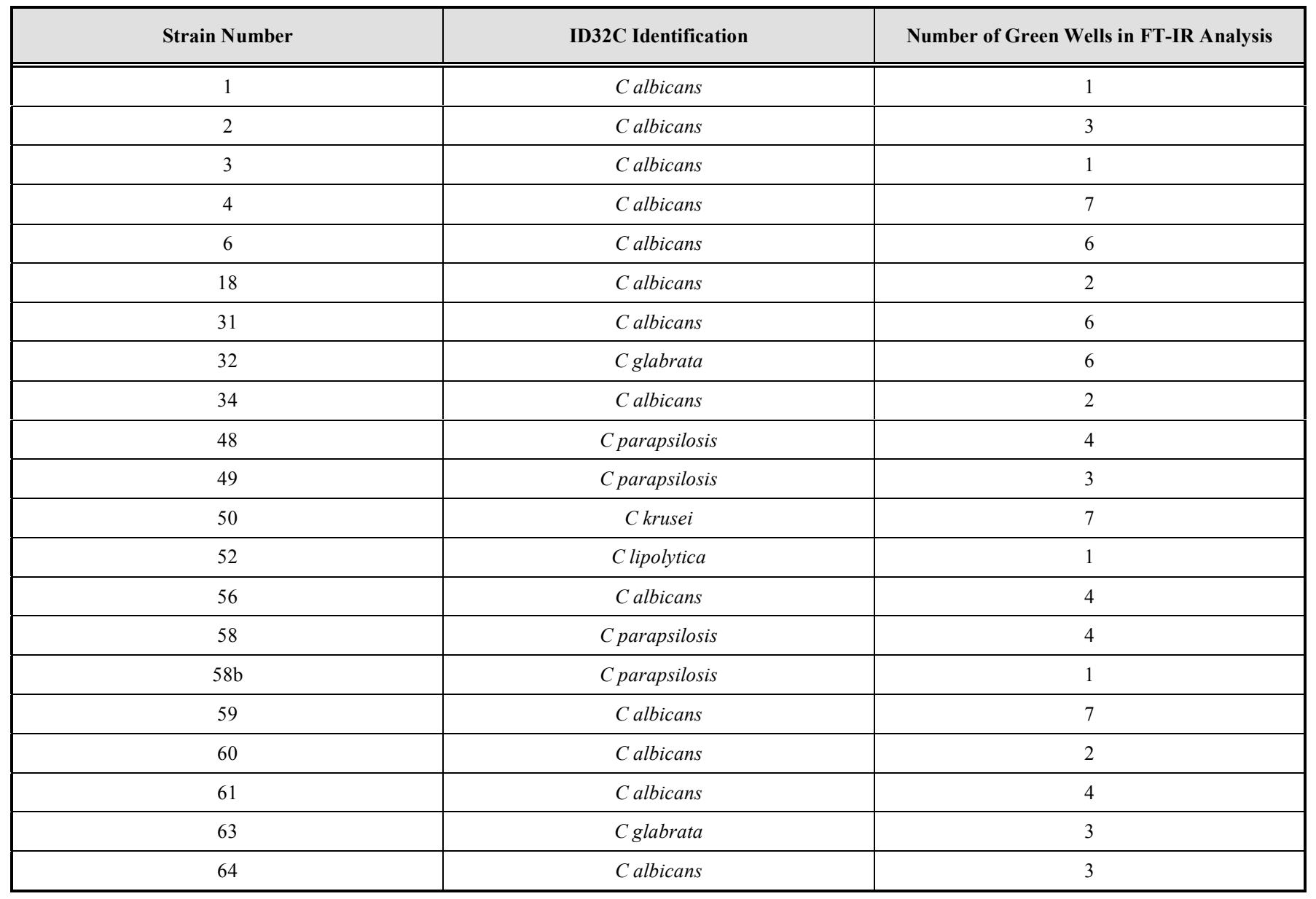




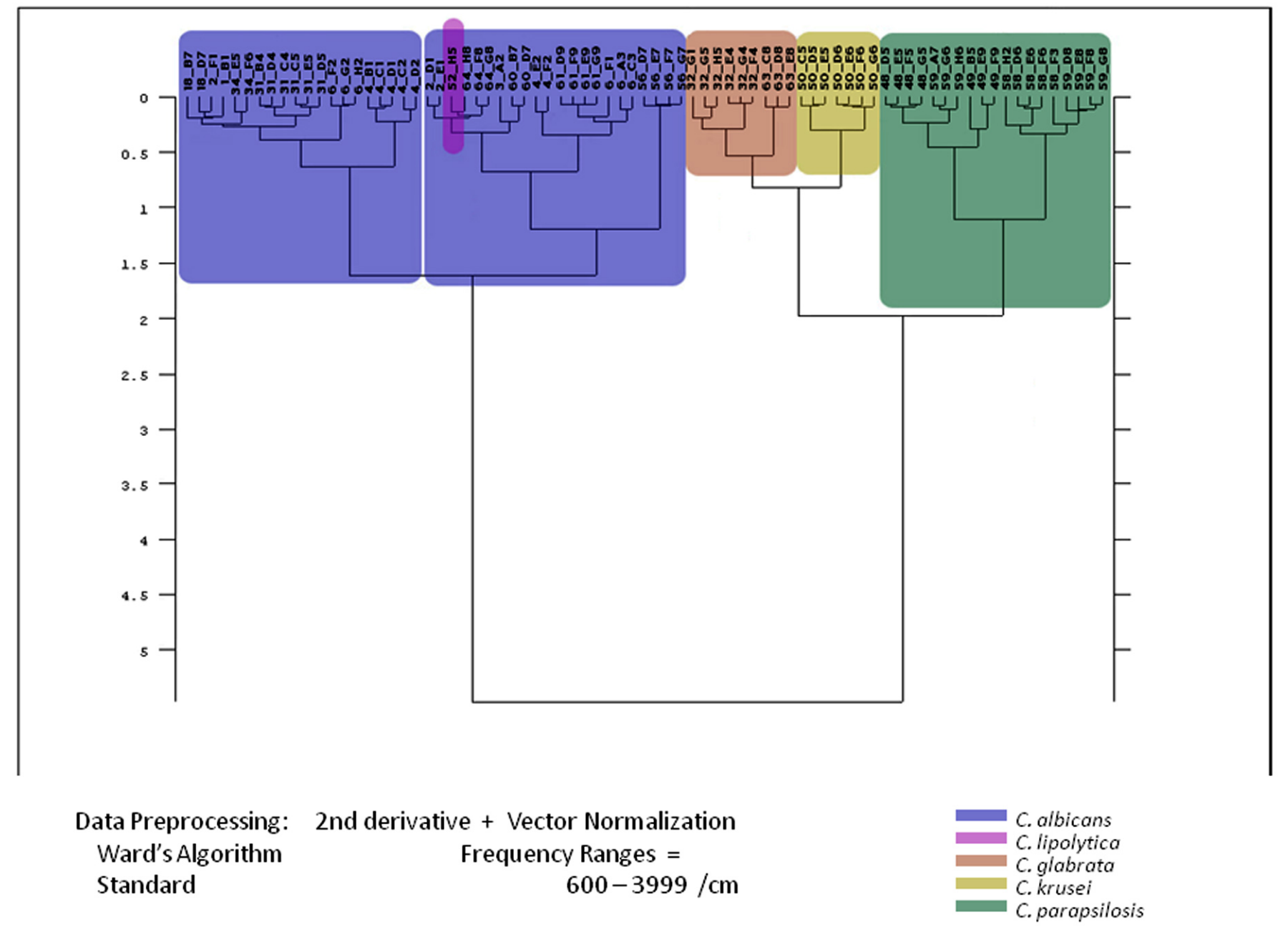

Fig. (1). Dendrogram of all 21 strains with superimposed ID32C identification results.

misleading in some cases.

Also puzzling was the fact that $C$ albicans was divided into two clusters that differed by as much as other species differed. This is conflictive with the results obtained by Essendoubi, M. et al. [5] and in a blind trial we would wrongly consider two strains, one from each of these two clusters, as different species when in fact they are not.

We conclude that FT-IR is an easy and inexpensive technique to fast-screen strains of yeasts and potentially identify them or even type them in suspicion of an outbreak, as it speeds analysis because it is not necessary to wait for cultures to grow during biochemical based identification. The equipment however is very expensive and at least 7 replicates should be welled in order to obtain at least one approved reading. This means that a 96 well plate allows a through-put of only 13 strains. Also FT-IR reproducibility depends on culture conditions, so reference strains should be used in the analysis batch in order to bridge results with previous tests.

Recently however, Roscini, L. et al. [1] give way to a more consistent approach with the selection of the most variable sections of IR spectra combined with a minimum amount of 5 replicas for consistency. Since our goal of using this method routinely is to determine rapidly the relatedness of strains when confronted with a possible outbreak more than for identification itself, we will carry on using this method following Roscini, L. et al.'s results [1].

\section{ACKNOWLEDGEMENT}

None declared.

\section{CONFLICT OF INTEREST}

None declared.

\section{REFERENCES}

[1] L. Roscini, L. Corte, L. Antonielli, P. Rellini, F. Fatichenti and G. Cardinali, "Influence of cell geometry and number of replicas in the reproducibility of whole cell FTIR analysis", Analyst, vol. 135, pp. 2099-2015, 2010.

[2] A. Alves, I. Henriques, A. Santos, M. Tacão and A. Correia, "Tipagem genética em microrganismos", M.S. in Molecular Microbiology, University of Aveiro, Aveiro, Portugal, 2003.

[3] J. Guarro, J. Gené and A. M. Stchigel, "Developments in fungal taxonomy", Clin. Microbiol. Res., vol. 12, no. 3, pp. 454-500, July 1999.

[4] E. M. Timmins, S. A. Howell, B. K. Alsberg, W. C. Noble and R. Goodacre, "Rapid differentiation of closely related candida species and strains by pyrolysis-mass spectrometry and fourier transform- 
infrared spectroscopy", J. Clin. Microbiol., vol. 36, pp. 367-374, February 1998.

[5] M. Essendoubi, D. Toubas, M. Bouzaggou, J. M. Pinon, M. Manfait and G. D. Sockalingum. "Rapid identification of Candida species by FT-IR microspectroscopy", Biochimica et Biophysica Acta, vol. 1724, pp.239-247, August 2005.

[6] C. Sandt, G. D. Sockalingum, D. Aubert, H. Lepan, C. Lepouse, M. Jaussaud, A. Leon, J. M. Pinon, M. Manfait and D. Toubas, "Use of fourier-transform infrared spectroscopy for typing of candida albicans strains isolated in intensive care units", J. Clin. Microbiol., vol. 41, pp. 954-959, March 2003.

[7] J. Perlroth, B. Choi and B. Spellberg, "Nosocomial fungal infections: epidemiology, diagnosis, and treatment", Med Mycol., vol. 45, no. 4, pp. 321-346, June 2007.

CC Brandão et al.; Licensee Bentham Open.

This is an open access article licensed under the terms of the Creative Commons Attribution Non-Commercial License (http://creativecommons.org/licenses/by-nc/ 3.0/) which permits unrestricted, non-commercial use, distribution and reproduction in any medium, provided the work is properly cited. 